\title{
Spatiotemporal Cellular Networks Maintain Immune Homeostasis in the Lung
}

Authors:

Disclosure:

Acknowledgements:

Received:

Accepted:

Keywords:

Citation:

\section{Jessica G. Borger}

Central Clinical School, Monash University, Alfred Research Alliance, Melbourne, Australia

*Correspondence to jessica.borger@monash.edu

The author has declared no conflicts of interest.

The author thanks Layla Southcombe for the invitation to write a review for EMJ Respiratory, and thanks Dr Lakshanie Wickramasinghe for her critical reading of this manuscript.

03.07.20

05.09 .20

$\mathrm{y} \delta$-T cell, dendritic cell (DC), homeostasis, innate lymphoid cells (ILC), lung, macrophage, mucosal, T cell, regulatory T cell (Treg), Th1, Th2, Th17.

EMJ Respir. 2020;8[1]:108-119.

\section{Abstract}

A dynamic and intricately connected tissue-resident immune cell network continuously monitors the lungs, which are incessantly subjected to external environmental insults. The lungs are protected by the respiratory epithelium, which not only serves as a physical barrier through mucociliary mechanisms, but also a reactive one that can release cytokines, chemokines, and other defence proteins in response to danger signals. In the maintenance of pulmonary homeostasis in health, the lung-resident immune cell network instructs tolerance to innocuous particulates and can rapidly and efficiently drive immunity and memory to pathogenic antigens. This review examines the spatiotemporal dynamics that underlie the exquisite network of highly specialised immune cells and their mediators in the support of pulmonary tissue homeostasis and effective lung immunity in health. In particular, this review examines the specialised immune cells that reside in distinct populations within the diverse compartments of the lung, and the molecular signals that retain and recruit lungresident immune cells, to further our understanding of how these can be targeted therapeutically to return inflamed or diseased lungs to homeostasis.

\section{INTRODUCTION}

Lung homeostasis is maintained by an immune cell network of tissue-resident cells that constantly monitor the respiratory tract, which is exposed to inhaled matter containing aeroallergens and airborne pathogens that can cause infection, and noxious agents including dust, smoke, and other environmental pollutants known to induce lung tissue injury. Channelling air into the respiratory system are the conducting airways, comprised of a single layer of ciliated epithelium tissue interspersed with mucus-producing goblet cells that protects against particulates and infectious agents that adhere to the mucus, and through the actions of the cilia are cleared from the airways. This respiratory epithelial cell surface therefore represents a large and exquisitely delicate surface undergoing continuouschallengeby immunogenic antigens. Maintenance of pulmonary immune 
homeostasis, required to dynamically support efficient gas exchange between the epithelial cell surfaces and the vascular bed, is provided at this interface by constant immune surveillance differentiating between self and the environment, instructing tolerance mechanisms to innocuous inhaled particles, and through mounting rapid responses to pathogenic challenge.

Maintenance of immune homeostasis and the triggering of pathogenesis requires a sophisticated understanding of the dynamic interplay between the network of tissue-resident immune cells within the microanatomical organisation of the lung. This review will discuss the spatiotemporal cellular dynamics of the innate and adaptive immune cell networks during homeostasis, focussing on the participation of the individual lung-resident immune cell types and their intricate interplay to maintain the pulmonary microenvironment. How these immune cells then participate in wound healing and tissue repair, become dysregulated and contribute to chronic inflammation, and the treatment of subsequent respiratory complications will not be discussed in this review, but reference to topical studies on advancements in these fields will be highlighted.

\section{SPATIOTEMPORAL ORGANISATION OF THE PULMONARY IMMUNE CELL NETWORK}

The lung is populated by heterogenous tissueresident innate and adaptive immune cells that function together to maintain tissue homeostasis and protect from recurrent pathogenic challenges. Dysregulation of the immune response can cause acute respiratory distress syndrome ${ }^{1}$ and asthma, $^{2}$ or drive chronic inflammatory diseases ${ }^{3}$ including chronic obstructive pulmonary disease (COPD), ${ }^{4}$ bronchopulmonary dysplasia, ${ }^{5}$ pulmonary fibrosis, ${ }^{6}$ and lung cancer. ${ }^{7}$ To appreciate the maintenance of homeostasis and development of immune responses within the lung, it is critically important to consider spatiotemporally how and where immune cells interact and function.

In the steady state, macrophages, the predominant innate leukocyte population, and dendritic cells (DC) continually sample and phagocytose the majority of inhaled innocuous and pathogenic particulate material that enters the airways, to protect local tissues from damage by suppressing induction of the adaptive immune response. ${ }^{8}$ Cluster of differentiation (CD) $4+$ and CD8+ $T$ cells are the prevalent adaptive immune cells that generate specific effector and memory responses to innocuous bacterial, viral, and fungal antigens. ${ }^{9}$ Pathogenic antigen not cleared from the conducting airways within mucous secretions from goblet cells or digested by macrophages is sampled through the airway epithelium by DC and instead transported to the lung-draining lymph nodes for processing into the major histocompatibility complex Class I or II pathways for generation of a specific adaptive T-cell effector response (Figure 1).

Distinct leukocyte networks extend throughout the entirety of the lung, reflecting contrasting and variable levels of airborne antigen exposure experienced throughout the pulmonary system, which facilitate highly specific and directed immune responses to maintain tissue homeostasis. The lung is segregated into numerous anatomically distinct cellular compartments, including the conducting airway mucosa, the lung parenchyma, the draining lymph nodes, bronchoalveolar space, bronchus-associated lymphoid tissue (BALT), the intravascular cell pool, and periarterial space. ${ }^{10}$

\section{Conducting Airway Mucosa}

The conducting airway mucosa is composed of ciliated epithelial cells and mucus-secreting goblet cells that remove inhaled particulate through mucociliary clearance mechanisms. The bronchial epithelium contains a unique leukocyte network, enriched in DC, predominantly myeloid DC, as well as plasmacytoid DC (pDC) and pulmonary alveolar macrophages (AM). Lungresident airway mucosal DC are reported to be strategically positioned with highly motile projections extended between epithelial cells to support direct antigen sampling from the airway luminal surface.1-14 Interestingly, many of these studies are inferred from static imaging and flow cytometry studies ${ }^{11,15,16}$ with recent work utilising elegant slice imaging, suggesting the conducting airways are rarely sites of transepithelial DC projections, ${ }^{17}$ challenging current dogma and indicating antigenic sampling occurs upon escape of particulate from mucociliary actions and its exposure within the lung parenchyma. 


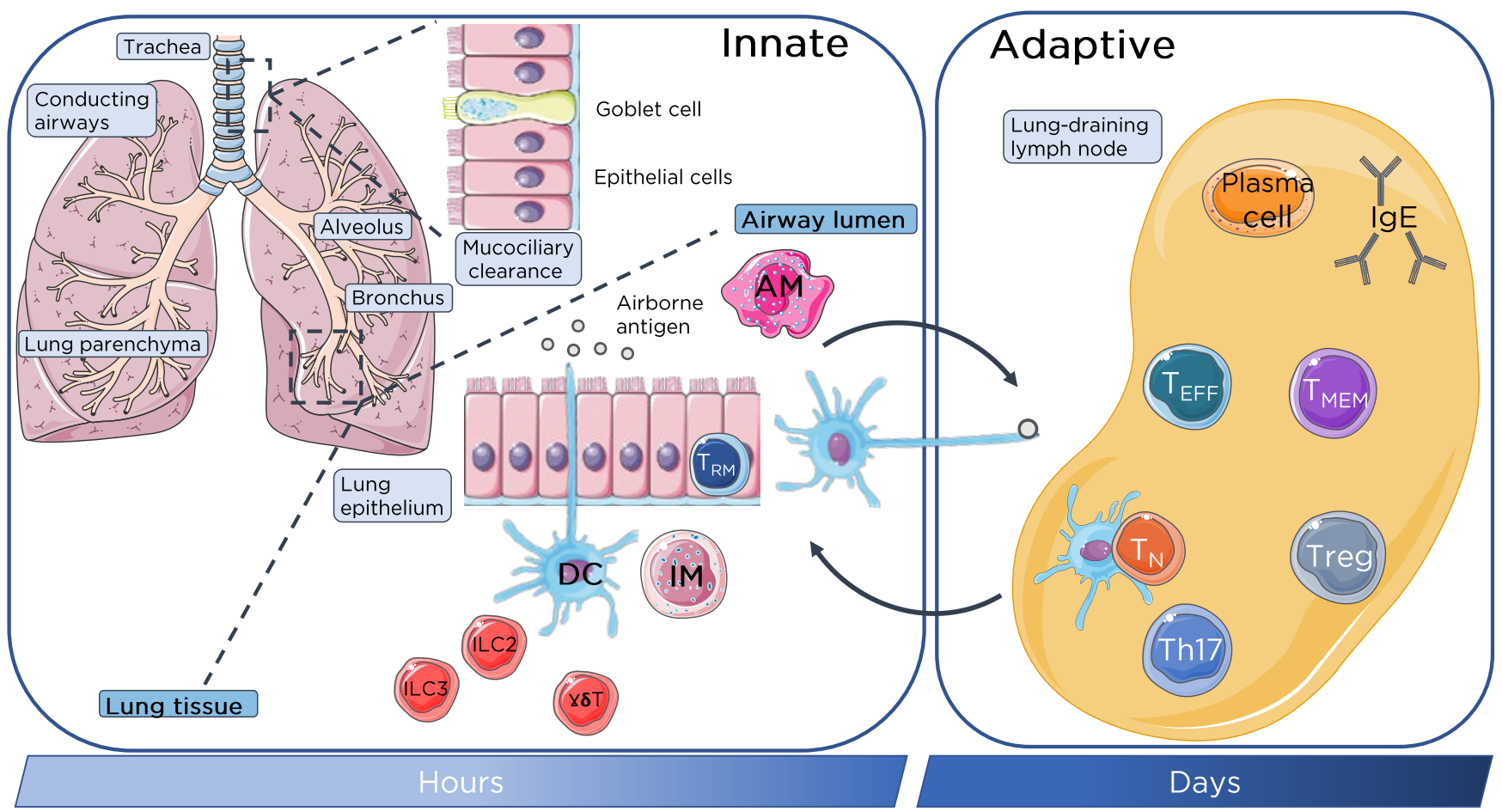

Time after antigen presentation

Figure 1: Lung-resident immune cell network.

Immune homeostasis is maintained in the numerous distinct compartments of the lung by specific populations of lung-resident immune cells beyond initial mucociliary clearance of inhaled innocuous and pathogenic antigens. Within the airways lung-resident AM and DC from the rapidly responding innate immune system phagocytose airborne antigen for clearance or processing and presentation respectively to $T$ cells in the lung-draining lymph nodes. These innate cells, along with ILC and innate-like $\gamma \delta$-T cells provide a rapid first-line of defence to inhaled antigenic particulates. Antigens escaping mucociliary or phagocytic clearance are sampled by DC through the surface lung epithelium of the bronchial mucosa or alveoli of the conducting airways. Activated DC migrate to the lung-draining lymph nodes through the lymphatics which are recognised by $T_{N}$ which become activated and differentiate into $T_{E F F}$ including Treg and Th17 or generate memory. Antigen-specific activate $T$ cells migrate through the lymphatics and pulmonary capillaries into the lung parenchyma and site of infection. B cells will also generate IgE antibody response through the differentiation and activation of plasma B cells.

AM: alveolar macrophages; DC: dendritic cells; IM: interstitial macrophages; ILC: innate lymphoid cells; $T_{E F F}$ : effector T cells; $T_{\text {MEM }}$ : memory $T$ cells; $T_{N}$ : naïve T cells; Treg: regulatory $T$ cells.

This figure was produced using images modified from Servier Medical Art (Servier, https://smart.servier.com/, licensed under a Creative Commons Attribution 3.0 Unported Licence).

Intraepithelial lymphocytes (IEL), specialised lymphocytes that do not require antigen priming for their effector functions, are also found in relatively high numbers in the mucosal linings in the lung as well as gastrointestinal tract, where they are components of the gut-associated lymphoid tissue. Both CD8 and CD4+ intraepithelial $\mathrm{T}$ cells are found intraepithelially and in the lamina propria, and express an effector cell phenotype (CD45RO+). Indeed, in allergic inflammation and bronchial infections, intraepithelial lymphocytes participate in crosstalk with the bronchial epithelium in the regulation of inflammatory processes $^{18}$ and present with many functions. ${ }^{19}$ The lamina propria also homes antibody-producing plasma cells $(\lg \mathrm{A}+)$ and some $\mathrm{B}$ cells which may contribute to local antigen presentation, as demonstrated in the lymph nodes that drain the lungs. ${ }^{20}$

\section{Lung Parenchyma}

The lung parenchyma includes the respiratory bronchi, which branch into bronchioles and terminal bronchioles, which extend into alveolar 
ducts and alveolar sacs. The pulmonary circulation, a dynamic network of capillaries that are in close contact with the alveolar space in the interstitium that separates the alveoli, localises a unique immune response within the parenchyma.

The alveolar space, and not the airways, appears to be the main site of particulate ingestion and movement to the airways. Intravital imaging has demonstrated that AM are the major phagocytes in the alveolar space and become immobile and phagocytic following antigen encounter, digesting all antigenic material that evades mucosal clearance. Lung-resident DC project dendrites into the alveoli to support antigen uptake, differentiating innocuous insults from dangerous ones, and trafficking to the airways to release antigen into the interstitium. ${ }^{17-H a v i n g}$ an abundant number of $A M$ residing in the terminal alveoli suggests that particulate will be transported upward by DC to the branching airways to the lung-draining lymph nodes resulting in local or systemic activation of T cells. ${ }^{21}$ Thus, DC uptake and presentation only appears to account for a fraction of antigen uptake at steady state, which is enhanced during immune responses. ${ }^{17}$ This appears to be driven not by a change in the ability of DC to gain access to the alveoli, but by a rapid influx of monocyte-derived DC into the alveoli during antigenic challenge.

Another less commonly considered tissueresident immune cell, the neutrophil, resides throughout the pulmonary vasculature of the parenchyma at steady state, sequestered at high numbers in both narrow and wide capillaries. In response to inflammatory challenge in models of lung injury, lung-resident neutrophils dramatically increase within the pulmonary capillaries, cluster, and extravasate rapidly. ${ }^{17,22-24}$ Recent imaging has suggested that neutrophils may actually be sequestered following a leading monocyte population that transmigrates into the interstitium immediately after lung injury. 22 This suggests that in homeostasis, the lungs are primed to rapidly respond to insults due to the abundant number of neutrophils trapped throughout the microvasculature that are primed to enter the lung and immediately degranulate.

\section{Lung-Draining Lymph Nodes}

Lung-draining lymph nodes are an essential component of the pulmonary immune cell network, a critical site for the development of adaptive immunity in infection and allergy, ${ }^{25}$ and the primary site for the induction of immunological memory. Migratory DC and AM, although participating in alternate transport pathways of antigen presentation, both migrate from the lung to the lung-draining lymph nodes to actively transfer antigen and activate effector T-cells responses. ${ }^{26}$ Whether the lung-draining lymph nodes serve to functionally integrate with the overall immune system and exactly how this cross-communication is regulated is yet to be fully understood.

\section{Intravascular Leukocyte Pool}

The intravascular leukocyte pool is contained within the endothelium of capillaries and venules of the lung. Although the intravascular leukocyte pool is critical for the range of immune reactions in the lung, it remains unclear whether intravascular leukocytes are a prerequisite for efficient immune reactions in the lung ${ }^{25,27,28}$ and how their numbers and migration into the lung parenchyma is regulated. ${ }^{10}$

\section{Bronchus-Associated Lymphoid Tissue}

Although only present during early childhood in healthy individuals, BALT is a potential inductive site contained with the greater airway mucosa. ${ }^{29}$ BALT, comprising discrete lymphoid-cell aggregates underlying a specialised epithelium, is part of the integrated mucosal immune system, facilitating the migration of lymphocytes to other mucosal sites. ${ }^{30}$ BALT can reappear in conditions of inflammation such as smoking. ${ }^{31}$ Inducible BALT from mice that lack other organised lymphoid tissue can generate protective immunity against pathogens, such as influenza virus, ${ }^{32}$ which suggests in early life that BALT may play a role in maintaining local immunological homeostasis when central lymphoid structures in the respiratory tract are functionally immature.

\section{Periarterial Space}

Periarterial space is located around the pulmonary arteries surrounded by lymphatic vessels and blood capillaries. The periarterial space was recently demonstrated to be involved in pulmonary host defense, ${ }^{33}$ yet whether innate or adaptive immunity is dominant within the periarterial space and the mechanisms driving immune cell infiltrate from the alveoli to the periarterial space is yet to be understood. 


\section{CELLULAR DYNAMICS OF THE IMMUNE NETWORK IN THE LUNG}

A dynamic dual phagocytic system involving AM and neutrophils is continually removing inhaled particulates, but activation and recruitment of adaptive immune cells to the site of infection is critical for elimination of innocuous microorganisms from the alveolar space. Immune cell recruitment to the lungs is 'unique' in that it has two separate circulatory systems: the bronchial arteries from the systemic circulation that nourishes the bronchial wall and the lowpressure pulmonary system that circulates through the lung parenchyma. ${ }^{34}$ The mucosal immune responses of the central airways are also integrated with those of distinct mucosal tissues through mucosal-associated lymphoid tissue. Lymphocytes in the lung have distinct imprinting of tissue-homing properties. Unlike naïve $T$ cells that express adhesion molecules and chemokine receptors that restrict their migration to lymphoid tissue, ${ }^{35}$ activated memory $T$ cells downregulate these lymphoid-tissue-homing receptors and upregulate tissue-specific adhesion molecules and chemokine receptors that target their migration to non-lymphoid tissues. ${ }^{36}$

The regulation of T-cell migration in the lung is yet to be fully understood but it has been shown in viral infection that memory $T$ cells mainly traffic and recirculate through the pulmonary capillaries and selectively accumulate in the lung parenchyma. ${ }^{37}$ During homeostasis, endothelial cells in the pulmonary vasculature express intercellular adhesion molecule 1 (ICAM-1) and P-selectin. Lymphocyte function-associated antigen 1(LFA-1)-ICAM 1 interactions have been demonstrated as the requisite interaction to retain and support egress of effector $C D 8+T$ cell from the pulmonary vasculature. ${ }^{38}$ Further interactions with P-selectin, through P-selecting glycoprotein ligand 1 (PSGL-1) expression by CD8 $T$ cells, further mediates the recruitment and retention of $T$ cells in the alveoli and lung parenchyma. In addition, chemokine interactions, such as C-C chemokine receptor type 5 (CCR5) and chemokine ligand 5 (CCL5), was shown during viral challenge to recruit memory $T$ cells to the parenchyma and also appear to play a role in tissue-specific lung homing. ${ }^{37}$ Taken together with integrin mechanics, this suggests that specific combinations of integrin expression and chemokine gradients are an important mechanism for retention of $T$ cells in the airways during homeostasis and pathogenesis.

To summarise the role of chemokine receptors expressed on immune cells in homeostasis in the lung is difficult, and largely inferred from studies on their role in the pathogenesis of lung diseases including COPD, ${ }^{39,40}$ asthma and allergic airway diseases, ${ }^{41-43}$ pulmonary fibrosis, ${ }^{44,45}$ and pulmonary infections. ${ }^{46,47}$ To determine the role of chemokine receptors, mice with a particular gene knockout, blocking antibodies, and specific inhibitors of chemokine receptors have been studied in different lung disease models which are comprehensively discussed in a review by Tomankova et al. ${ }^{48}$

\section{TISSUE-RESIDENT IMMUNE CELLS CONTRIBUTING TO PULMONARY HOMEOSTASIS}

\section{Alveolar and Interstitial Macrophages}

Alveolar and interstitial macrophages maintain pulmonary immune homeostasis via their intimate interactions with other lung-resident cells, specifically pulmonary epithelial cells. Expression of a broad range of surface receptors enables them to sense the environment and signal to local stromal cells in order to maintain immune homeostasis, but also to sense change within the inhaled environment. Macrophages sense change within the inhaled environment that can be activating (toll-like receptors [TLR] 2, 4, 6; IL-1 receptor; IFN-y receptor; and TNF receptor) or suppressive (CD2OO receptor, signal-regulatory protein a, mannose receptor, triggering receptor expressed on myeloid cells 2 , IL-10 receptor, transforming growth factor [TGF] $\beta$ receptor ${ }^{49}$ It is clear that close interaction and communication between airway epithelial cells and airway macrophages is vital for maintenance of immune homeostasis within the respiratory tract.

At least two distinct macrophage populations exist in the lung at homoeostasis, termed AM and interstitial macrophages (IM) that are characterised by their distinct locations and unique functions (Figure 1).50 Located in the airway lumen, $A M$ are characterised by high expression of CD206, CD11c, and SiglecF+, but 
lack CD11b, which is expressed by other tissue resident macrophages. ${ }^{51}$ IM conversely lack CD206 and SiglecF, are CD11b bright, express only low levels of CD11c, and reside in the lung parenchyma. ${ }^{51} \mathrm{IM}$ release cytokines associated with the adaptive immune response, such as IL-10, which exerts a regulatory role in the lung, whereas immunosuppressive highly phagocytic AM play a central role in maintaining immunological homoeostasis. ${ }^{52}$ Indeed, in steady state, AM are largely quiescent, downregulating the phagocytic receptor Macrophage 1 antigen, and secrete nitric oxide, prostaglandin endoperoxide 2 , and immunoregulatory cytokines IL-10 and TGF $\beta$ to prevent uncontrolled inflammation within the respiratory tract.

The renewal of AM populations in the steady state largely occurs by local precursor-cell proliferation, but during inflammation renewal occurs via incoming monocytes and is regulated through the $\mathrm{C}$ chemokine receptor type 2 (CCR2) and chemokine ligand 2 (CCL2) axis. ${ }^{53}$ Of note is the functional phenotype of recently recruited monocytes contrasts significantly with that of lung-resident AM. Monocytes recruited to the alveolar space during inflammation display a proinflammatory phenotype with elevate levels of steady-state TNFa mRNA; increased neutrophil chemoattractants macrophage inflammatory protein 2 (MIP2), keratinocyte chemoattractant, and IFNy-induced protein 10 (CXCL10); TLR4 upregulation; and the expression of various lysosomal cysteine proteases involved in extracellular matrix degradation and tissue remodelling processes. ${ }^{54}$ Specifically, monocytes can function as efficient antigenpresenting cells (APC) before their maturation into immunosuppressive AM, which occurs over a period of days..$^{55}$ Interestingly, these 'converted' AM, which become increasingly similar to lung-resident AM, can persist for over 1 year after lung injury and cause profibrotic changes. ${ }^{56}$ The recruited monocyte population is heterogeneous, including immature DC, which will also enhance T-cell responsiveness through exposure to granulocyte-macrophage colony-stimulating factor. ${ }^{57}$

\section{Lung-Resident Dendritic Cells}

In the steady state, lung-resident DC are critically involved in antigen uptake and processing in the lung but lack the capacity for efficient antigen presentation until they migrate to the lung-draining lymph nodes.58 However, during inflammation, alveolar myeloid DC (AMDC) can become potent APC. ${ }^{59}$ AMDC present with a phenotypic profile of high expression of major histocompatibility complex Class II and CD205, together with low expression of CD8, CD40, CD80, and CD86, similar to systemic DC. Other distinct subpopulations of lung DC have also been identified ${ }^{60,61}$ within the airway mucosa but the main focus during inflammation is attributed to the AMDC pulmonary network. AMDC have a high turnover rate in the steady state, constantly transporting antigens from the mucosa to the lung-draining lymph nodes. ${ }^{62}$

During lung homeostasis, resident conventional $D C$ (CDC) reside in the lung as two phenotypically and functionally distinct subsets: CD11b+CD103- (cDC2) and CD11b-CD103+ $(\mathrm{CDC1}){ }^{63,64} \mathrm{CDC1}$ are located in the epithelial layer, extend their dendrites between epithelial cells to capture antigens in the airway lumen, and migrate to the lung-draining lymph nodes to cross-present to CD8+ T cells during respiratory viral infection. ${ }^{65} \mathrm{CDC} 2$ are located in the lamina propria and unable to directly penetrate the airway lumen and migrate to the lung-draining lymph nodes in a later stage of infection, shown to coincide with the peak of viral load in the lung tissue. ${ }^{65}$

pDC in the lungs play an immunoregulatory role by influencing regulatory $T$ (Treg) cells ${ }^{66}$ and have recently been implicated in tolerance induction to inhaled antigen. ${ }^{67,68}$ Furthermore, the high capacity of $\mathrm{pDC}$ to produce IFNa in response to microbial stimuli suggests this subset of DC may play a critical role in the lung in antiviral immunity. ${ }^{69-73}$ However, it is unlikely that pDC migrate out of lung tissues, but instead enter the lung-draining lymph node from the blood to respond and participate in local inflammation.

\section{Tissue-Resident Memory Cells}

The majority of lung $T$ cells are non-circulating tissue-resident memory T cells $\left(T_{R M}\right)$, which persist in stable frequencies for decades of human life. Lung $T_{R M}$ were first identified as CD4+ T cells that were retained specifically in the lung in parabiosis studies, occupying specific airway niches. ${ }^{74,75}$ Displaying a unique transcription profile ${ }^{76,77}$ and resident in specific mucosal, barrier, and 
lymphoid tissues, $T_{R M}$ were distinguished as a distinct subset of effector memory $T$ cells residing within the epithelium (Figure 1). $T_{R M}$ are sufficient to generate local inflammation, even in the absence of $\mathrm{T}$ memory cells from secondary lymphoid organs. ${ }^{78}$ Present in the healthy lung, $\mathrm{T}_{\mathrm{RM}}$ cells express CD69 and CD103 to promote tissue retention, as well as a diverse T-cell receptor repertoire. ${ }^{79,80}$ Recent studies reveal an important role for lung $T_{R M}$ in rapid protective immune response to maintain tissue homeostasis upon exposure to a diverse range of inhaled antigenic material, and are also important in surveillance for tumours and persistent viruses. Mouse studies have revealed the central role of $T_{R M}$ in multiple aspects of lung immunity including following influenza or respiratory syncytial virus infection ${ }^{81-83}$ and in response to allergen exposure, ${ }^{84,85}$ indicating high therapeutic potential.

\section{Regulatory T Cells}

Treg appear to be present from birth with their inducible phenotype influenced by local microbiota from early life. ${ }^{86}$ Lung-resident Treg are central for the maintenance of immune tolerance to airborne antigen ${ }^{87}$ and in the control of peripheral T-cell responses. ${ }^{88}$ Crosstalk between $A M$ and $T$ cells is considered a mechanism through which inducible Treg cells are generated and allergic responses are dampened. ${ }^{89}$ Treg cells have been shown as critical for lung homeostasis with adoptive transfer of inducible Treg cells inhibitingallergic inflammationand hyperreactivity via production of IL-10.90 Treg cells were also shown to attenuate airway hyperresponsiveness through recruitment to the airway mucosa following the first wave of inflammation triggered by allergen inhalation, demonstrating a critical role of Treg cells in homeostasis to maintain healthy pulmonary function. ${ }^{34}$

\section{T Helper 17 Cells}

T helper (Th)17 cells provide critical immunoregulation in the lung through the production of IL-17, IL-22, and IL-23. Although the role for Th17 cells in lung immune homeostasis remains poorly understood, Th17-derived IL-17 plays a critical response in early inflammation and pathogen clearance in the lung by mobilising neutrophils. Th17 cells are induced through the production of IL-6, IL-21, and TGF $\beta$ in mice and IL-6 and IL-1 in humans. Interestingly, Th17 and
Treg cell differentiation in the lung appears to be mutually exclusive with TGF $\beta$ inducing Th17 and Treg cells, although IL- 6 inhibits Treg-cell differentiation enabling the Th17 population. ${ }^{91,92}$ It has been proposed that the reversal of Treg cell suppressive function that is induced by TLR activation may be a reflection of an increase in Th17 cell differentiation (involving IL-6 and TGF $\beta$ ) rather than a decrease in the ability for suppression, ${ }^{91}$ suggesting a role for Th17 cells in re-establishing homeostasis.

\section{Innate Lymphoid Cells}

Innate lymphoid cells (ILC) constitute several phenotypically distinct groups of innate lymphocytes that lack the usual lineage markers that define conventional cells, notably antigenspecific receptors thus ILC do not mediate antigen-specific responses..$^{93}$ ILC numbers are largely tissue-resident, enhanced at barrier surfaces, and thought to be vital for maintenance of tissue homeostasis, repair, and regulation of immunity (reviewed in Borger et al. ${ }^{94}$ ). ${ }^{95}$ Like effector $T$ cells, ILC have been classified according to the transcription factors that they express and the effector cytokines that they secrete ILC1 (T-bet), ILC2 (Gata3), ILC3 (Roryt) ${ }^{96}$ and can rapidly response to changes in the local tissue microenvironment.

ILC2 are the main population of ILC within the lung, shown to direct protective immunity during helminth infections: development of allergic inflammation, tissue repair, and maintaining metabolic homeostasis. ${ }^{93,95,97}$ In an elegant series of parabiotic experiments using the ILC2 found within the lung were demonstrated to be of host origin, indicating residency. ${ }^{98}$ Moreover, virtually all of the ILC2 and ILC1 identified were situated within the lung parenchyma rather than the circulation, with ILC2 being the major population. Somewhat surprisingly, even during episodes of inflammation such as during helminth infection, expansion of ILC2 was largely due to local proliferation rather than recruitment. ${ }^{98}$ ILC2 accumulation in the lung can be facilitated by interaction with both local stromal cells as well as haematopoietic cells. Cytokines secreted by pulmonary epithelial cells such as IL-33 and TGF $\beta$ enhance the accumulation of ILC2 cells within the lung, particularly after encounter with allergen..$^{99,100}$ Interestingly, the majority of studies to date focus on ILC2, however, ILC3 are in fact 
the most prevalent group in the human lung and their rapid secretion of IL-17A and IL-22 has led to their investigation in inflammatory and infectious diseases. ${ }^{101}$ Interestingly, although the importance of ILC3 as a source of granulocytemacrophage colony-stimulating factor, an important cytokine in pulmonary host defence, in the lung is unknown, ILC3 in the gut are known to orchestrate inflammation.

\section{yס-T Cells}

yठ-T cells constitute $8-20 \%$ of resident pulmonary lymphocytes, where they respond to danger signals and facilitate orchestration of immune responses. ${ }^{102}$ The abundance of $\mathrm{V} \delta$ - T cells maintains lung tissue homeostasis. The lung is a major site for homing of $y \delta-T$ cells during in the perinatal period, with $\mathrm{V}_{\mathrm{y}} \sigma^{+} \mathrm{y} \delta-\mathrm{T}$ cells the major үठ-T cell population from birth until 8-10 weeks of age, whereas ${\mathrm{V} Y 4^{+}}^{+} \delta-T$ cells predominate from that age on. ${ }^{103}$ In adult mice, $y \delta-T$ cells are divided into subsets expressing $\mathrm{V}_{\gamma} 4^{+}(45 \%), \mathrm{V}_{\gamma} 1^{+}$(15\%), $\mathrm{V}_{\gamma} 6^{+}(20 \%)$, and $\mathrm{V}_{\mathrm{V}} 7^{+}$(rare). ${ }^{104,105}$ These $\mathrm{y} \delta$-T cells are present in all regions of the lung, except for the airway mucosa. Interestingly, $\mathrm{V}_{\mathrm{Y}} 4^{+}$and $\mathrm{V}_{\mathrm{Y}} \mathrm{1}^{+}$ populations have a more parenchyma-biased distribution. ${ }^{105}$ Evidence of lymphoid precursors present in the lungs indicates that these cells might undergo differentiation and selection in the lung environment.

The $y \delta-T$ cells residing in the lung are potent producers of IL-17 whereas $y \delta-T$ cells expressing a different T-cell receptor in the skin, gut, liver, spleen, uterus, and peripheral blood can produce IL-17 or IFN-y. . $^{106} \mathrm{IL}-17$ producing cells, such as ILC3, yठ-T cells, natural killer T cells, mucosal associated invariant T cells, and ILC3, as well as adaptive Th17 cells, play distinct roles in host defence against diverse pathogens. ${ }^{107}$ The abundance of $\gamma \delta-T$ cells in the lung supports tissue homeostasis, through potentially a similar IL-17/IL-22 axis as ILC3, although $\gamma \delta-T$ cells have also been shown to play critical roles in bacterial clearance and the prevention of inflammation and lung fibrosis..$^{108}$

In the lung, although little is known of the role of $\gamma \delta-T$ cells in maintaining pulmonary homeostasis, $\bar{\delta}-T$ cells rapidly respond to pathogens as critical effector cells in innate host responses. ${ }^{109}$ This now introduces the question of how $y \delta-T$ cells, along with other tissue-resident unconventional $\mathrm{T}$ cells (mucosal associated invariant $\mathrm{T}$ cells, natural killer T cells) functionally integrate with ILC to enhance the innate-like immune response (reviewed in Borger et al. ${ }^{110}$ ). Whether or not these innate-like lymphocytes coregulate one another or the adaptive T-cell arm, or function independently remains to be answered. Their enhanced sensitivity to the changing lung tissue microenvironment and rapid innate ability to respond to diverse antigens, in participation with the almost immediate ability of macrophages and neutrophils to phagocytose and degranulate and the highly specific response of the adaptive arm and generation of memory, demonstrates a highly complex spatiotemporal network exists in the lung immune system to maintain tissue homeostasis and protect from lung injury.

Together the cells of the innate and adaptive immune system provide diverse effector and regulatory mechanisms that maintain pulmonary homeostasis during the persistent challenges of aeroallergens, airborne pathogens, and noxious agents along the respiratory tract (Figure 2). The dysregulation of the exquisite balance between effector and regulatory immune processes is an important disease mechanism contributing to chronic lung disease such as COPD and asthma; ${ }^{111112}$ in viral, fungal, helminthic, and other pathogenic infections; ${ }^{113}$ and in the development of lung cancer. ${ }^{114}$ COPD, for example, is a pathologically complex disease with patients presenting with inflamed airways containing macrophages, neutrophils, DC, and CD8 T cells. The influx of these inflammatory effector cells and the activation of tissue-resident cells including ILC and $y \delta-T$ cells drive airway remodelling and parenchymal destruction through the secretion of inflammatory mediators such as reactive oxygen species; chemokines including IL-8, proinflammatory cytokine TNFa, IL-1 $\beta, I L-6$, and IL-17; and proteases including matrix metalloproteinase and neutrophil elastase. Cigarette smoke, an established risk factor for COPD, has been shown to have a suppressive effect on innate immune cells within the respiratory tract and cause defects in the generation of adaptive immunity in the lung..$^{115,116}$ In this respect, although the immune cells that maintain tissue homeostasis and pulmonary function are still abundantly present, they are functionally deficient or abnormal. 


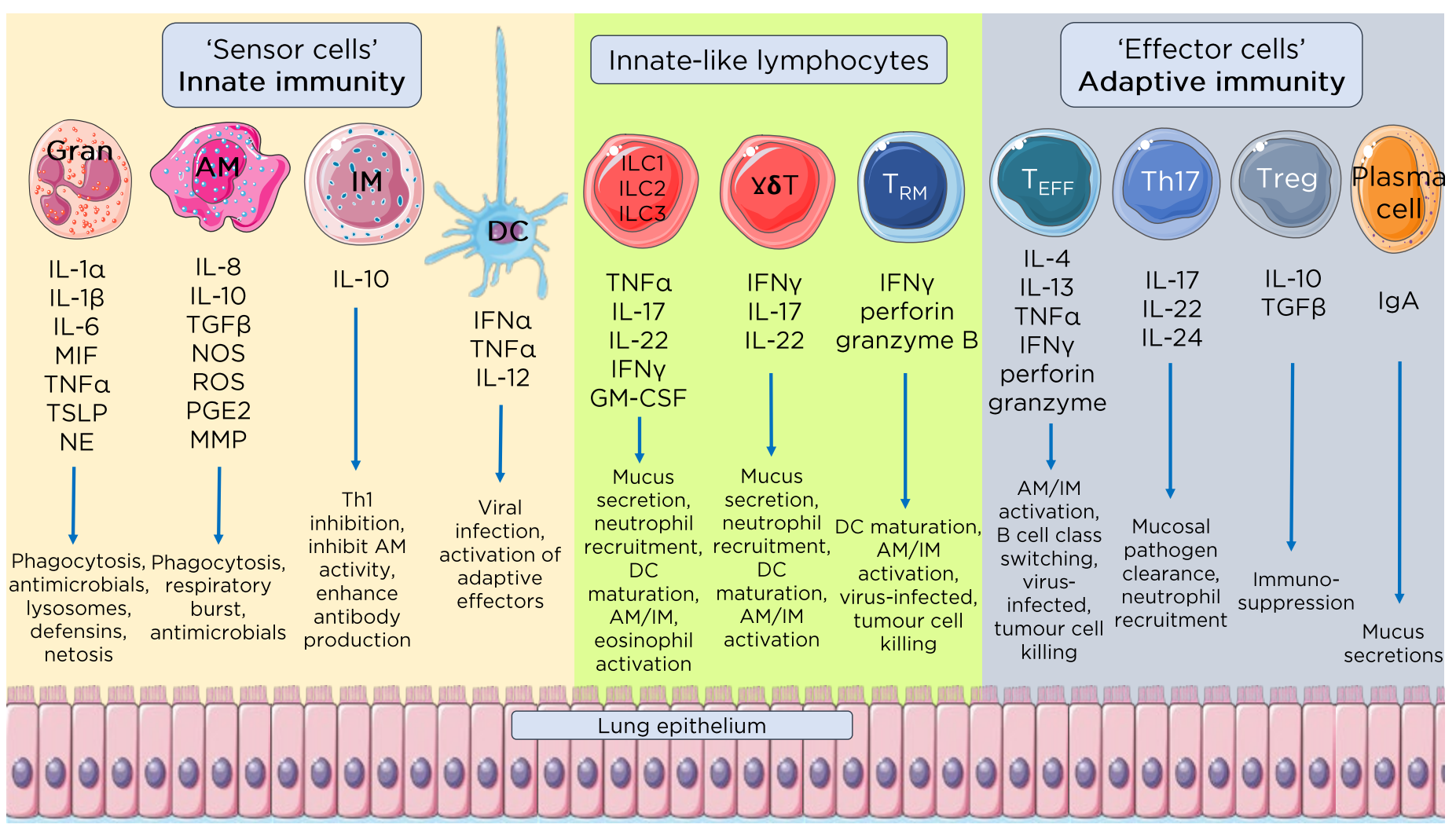

Figure 2: Effector and regulatory mechanisms that maintain pulmonary homeostasis.

Immune homeostasis in the lung requires an exquisite balance between effector cytokine and soluble factors with regulatory cytokines and humoral responses. The immediate innate response made of 'sensor cells' including granulocytes such as neutrophils and eosinophils, AM, IM, and DC, continually sample and respond to inhaled antigens to clear localised infections. This first and immediate line of immune defence secrete various factors such as IFN to clear pathogens, and other proinflammatory cytokines including IL-1, IL-6, and TGFB. Tissue-resident innate-like lymphocytes, including ILC, $\mathrm{y} \delta-\mathrm{T}$ cells, and $\mathrm{T}_{\mathrm{RM}}$ cells, bridge the innate and adaptive immune systems, secreting proinflammatory mediators and signals to recruit and activate effector cells from the adaptive immune system. Together these cytokines and soluble mediators serve to promote pathogen clearance, tissue repair and the maintenance of pulmonary immune homeostasis.

AM: alveolar macrophages; DC: dendritic cells; GM-CSF: granulocyte-macrophage colony-stimulating factor; Gran: granulocytes; IM: interstitial macrophages; MIF: macrophage migration inhibitory factor; MMP: matrix metallopeptidase; NE: neutrophil elastase; NOS: nitric oxide synthase; PGE2: prostaglandin E2; ROS: reactive oxygen species; $T_{E F F}$ : effector T cells; TGFB: transforming growth factor $\beta$; Th17: T helper 17; Treg: regulatory $T_{\text {cells; }} T_{R M}$ : tissue-resident memory T cells; TSLP: thymic stromal lymphoprotein.

This figure was produced using images modified from Servier Medical Art (Servier, https://smart.servier.com/, licensed under a Creative Commons Attribution 3.0 Unported Licence).

Therefore, it could be surmised that in chronic lung disease, and other lung pathologies, the immune sentinels responsible for pulmonary immune homeostasis have been detrimentally reprogrammed.

\section{CONCLUDING REMARKS}

Numerous immune cell populations contribute to the maintenance of pulmonary immune homeostasis, and in many cases exhibit phenotypic and functional features that appear specifically adapted to the unique local lung microenvironments. The immune cell network provides efficient surveillance in the lung, discriminating between innocuous and potentially pathogenic antigens due to the unique combination and spatiotemporal localisation of immune cells residing in the microanatomically distinct lung compartments.

To fully appreciate the maintenance of lung homeostasis, a greater understanding of the 
regulation of leukocyte influx into the distinct lung compartments is still required. In particular it is still largely unknown how these immune cells directly interact with tissue-resident mucosal cells, and what directs migration between the distinct lung compartments to maintain pulmonary homeostasis. By understanding the spatiotemporal kinetics and organisation of the immune cell network in the lung, cells or process in specific compartments can be targeted therapeutically to return inflamed or diseased lungs to homeostasis.

\section{References}

1. Rubenfeld GD et al. Incidence and outcomes of acute lung injury. N Engl J Med. 2005;353(16):1685-93.

2. Borish L. The immunology of asthma: asthma phenotypes and their implications for personalized treatment. Ann Allergy Asthma Immunol. 2016;117(2):108-14.

3. Marsland BJ et al. Immune system dysregulation in chronic lung disease. Eur Respir J. 2011;38(3):500-1.

4. Bhat TA et al. Immune dysfunction in patients with chronic obstructive pulmonary disease. Ann Am Thorac Soc. 2015;12(Suppl 2):S169-75.

5. Wickramasinghe LC et al. Lung and eye disease develop concurrently in supplemental oxygen-exposed neonatal mice. Am J Pathol. 2020;190(9):1801-12.

6. Kolahian $\mathrm{S}$ et al. Immune mechanisms in pulmonary fibrosis. Am J Respir Cell Mol Biol. 2016;55(3):309-22.

7. Domagala-Kulawik J. The role of the immune system in non-small cell lung carcinoma and potential for therapeutic intervention. Transl Lung Cancer Res. 2015;4(2):177-90.

8. MacLean JA et al. Sequestration of inhaled particulate antigens by lung phagocytes. A mechanism for the effective inhibition of pulmonary cell-mediated immunity. Am J Pathol. 1996;148(2):657-66.

9. Chen K, Kolls JK. T cell-mediated host immune defenses in the lung. Annu Rev Immunol. 2013;31:605-33.

10. Tschernig T, Pabst R. What is the clinical relevance of different lung compartments? BMC Pulm Med. 2009;9:39.

11. Lambrecht BN, Hammad H. Biology of lung dendritic cells at the origin of asthma. Immunity. 2009;31(3):412-24.

12. van Rijt LS et al. In vivo depletion of lung CD11c+ dendritic cells during allergen challenge abrogates the characteristic features of asthma. J Exp Med. 2005;201(6):981-91

13. Veres TZ et al. Spatiotemporal and functional behavior of airway dendritic cells visualized by twophoton microscopy. Am J Pathol. 2011;179(2):603-9.

14. Vermaelen KY et al. Specific migratory dendritic cells rapidly transport antigen from the airways to the thoracic lymph nodes. J Exp Med. 2001;193(1):51-60.

15. Jakubzick $C$ et al. Lymph-migrating, tissue-derived dendritic cells are minor constituents within steadystate lymph nodes. J Exp Med. 2008;205(12):2839-50.

16. Jakubzick $\mathrm{C}$ et al. Optimization of methods to study pulmonary dendritic cell migration reveals distinct capacities of DC subsets to acquire soluble versus particulate antigen. J Immunol Methods. 2008:337(2):121-31.

17. Thornton EE et al. Spatiotemporally separated antigen uptake by alveolar dendritic cells and airway presentation to $T$ cells in the lung. $J$ Exp Med. 2012;209(6):1183-99.

18. Gebb SA et al. Sites of leukocyte sequestration in the pulmonary microcirculation. J Appl Physiol (1985). 1995:79(2):493-7.

19. Lien DC et al. Physiological neutrophil sequestration in the lung: visual evidence for localization in capillaries. J Appl Physiol (1985). 1987;62(3):1236-43.

20. Lund FE et al. B cells are required for generation of protective effector and memory CD4 cells in response to Pneumocystis lung infection. J Immunol. 2006;176(10):6147-54.

21. Lelkes E et al. The spatiotemporal cellular dynamics of lung immunity. Trends Immunol. 2014;35(8):379-86.

22. Kreisel D et al. In vivo two-photon imaging reveals monocyte-dependent neutrophil extravasation during pulmonary inflammation. Proc Natl Acad Sci U S A. 2010;107(42):18073-8.

23. Looney MR et al. Stabilized imaging of immune surveillance in the mouse lung. Nat Methods. 2011;8(1):91-6.

24. Kreisel $D$ et al. Emergency granulopoiesis promotes neutrophildendritic cell encounters that prevent mouse lung allograft acceptance. Blood. 2011;118(23):6172-82

25. Pabst R, Tschernig T. Lymphocytes in the lung: an often neglected cell. Numbers, characterization and compartmentalization. Anat Embryol (Berl). 1995;192(4):293-9.

26. Kirby $\mathrm{AC}$ et al. Alveolar macrophages transport pathogens to lung draining lymph nodes. J Immunol. 2009;183(3):1983-9.

27. Winkler GC. Review of the significance of pulmonary intravascular macrophages with respect to animal species and age. Exp Cell Biol. 1989;57(6):281-6.

28. Gill SS et al. Role of pulmonary intravascular macrophages in endotoxin-induced lung inflammation and mortality in a rat model. Respir Res. 2008;9:69.

29. Tschernig T et al. Bronchusassociated lymphoid tissue (BALT) in the lungs of children who had died from sudden infant death syndrome and other causes. Thorax. 1995;50(6):658-60.

30. Brandtzaeg P, Pabst R. Let's go mucosal: communication on slippery ground. Trends Immunol. 2004;25(11):570-7.

31. Tschernig T, Pabst R. Bronchusassociated lymphoid tissue (BALT) is not present in the normal adult lung but in different diseases. Pathobiology. 2000;68(1):1-8.

32. Moyron-Quiroz JE et al. Role of inducible bronchus associated lymphoid tissue (iBALT) in respiratory immunity. Nat Med. 2004;10(9):927-34

33. Reppe $\mathrm{K}$ et al. Immunostimulation with macrophage-activating lipopeptide-2 increased survival in murine pneumonia. Am J Respir Cel Mol Biol. 2009;40(4):474-81.

34. Holt PG et al. Regulation of immunological homeostasis in the respiratory tract. Nat Rev Immunol. 2008;8(2):142-52.

35. Cose $\mathrm{S}$ et al. Evidence that a significant number of naive $T$ cells enter non-lymphoid organs as part of a normal migratory pathway. Eur J Immunol. 2006;36(6):1423-33.

36. Agace WW. Tissue-tropic effector $T$ cells: generation and targeting opportunities. Nat Rev Immunol. 2006;6(9):682-92.

37. Kohlmeier JE et al. The chemokine receptor CCR5 plays a key role in the early memory CD8+ T cell response to respiratory virus infections. Immunity. 2008;29(1):101-13.

38. Galkina $E$ et al. Preferential migration of effector CD8+ T cells into the interstitium of the normal lung. J Clin Invest. 2005;115(12):3473-83. 
39. Caramori G et al. Chemokines and chemokine receptors blockers as new drugs for the treatment of chronic obstructive pulmonary disease. Curr Med Chem. 2013;20(35):4317-49

40. Donnelly LE, Barnes PJ. Chemokine receptors as therapeutic targets in chronic obstructive pulmonary disease. Trends Pharmacol Sci. 2006:27(10):546-53.

41. Lloyd CM, Brown Z. Chemokine receptors : therapeutic potential in asthma. Treat Respir Med. 2006;5(3):159-66.

42. Lukacs NW et al. Chemokine receptors in asthma: searching for the correct immune targets. J Immunol. 2003;171(1):11-5.

43. Palmqvist $\mathrm{C}$ et al. Chemokines and their receptors as potential targets for the treatment of asthma. $\mathrm{Br} \mathrm{J}$ Pharmacol. 2007:151(6):725-36.

44. Strieter RM et al. CXC chemokines in angiogenesis relevant to chronic fibroproliferation. Curr Drug Targets Inflamm Allergy. 2005;4(1):23-6.

45. Strieter RM et al. The role of CXC chemokines in pulmonary fibrosis. $J$ Clin Invest. 2007;117(3):549-56

46. Oldstone MB et al. Dissecting influenza virus pathogenesis uncovers a novel chemical approach to combat the infection. Virology. 2013:435(1):92-101.

47. Slight SR, Khader SA. Chemokines shape the immune responses to tuberculosis. Cytokine Growth Factor Rev. 2013;24(2):105-13.

48. Tomankova T et al. Chemokine receptors and their therapeutic opportunities in diseased lung: far beyond leukocyte trafficking. Am J Physiol Lung Cell Mol Physiol. 2015;308(7):L603-18.

49. Hussell T, Bell TJ. Alveolar macrophages: plasticity in a tissuespecific context. Nat Rev Immunol. 2014;14(2):81-93.

50. Byrne AJ et al. Pulmonary macrophages: key players in the innate defence of the airways. Thorax. 2015;70(12):1189-96.

51. Gibbings SL et al. Three unique interstitial macrophages in the murine lung at steady state. Am J Respir Cell Mol Biol. 2017;57(1):66-76.

52. Bedoret $D$ et al. Lung interstitial macrophages alter dendritic cell functions to prevent airway allergy in mice. J Clin Invest. 2009:119 (12):3723-38.

53. Winter $\mathrm{C}$ et al. Lung-specific overexpression of CC chemokine ligand (CCL) 2 enhances the host defense to Streptococcus pneumoniae infection in mice: role of the CCL2-CCR2 axis. J Immunol. 2007;178(9):5828-38.

54. Maus UA et al. Resident alveolar macrophages are replaced by recruited monocytes in response to endotoxin-induced lung inflammation. Am J Respir Cell Mol Biol. 2006:35(2):227-35.

55. Bilyk N, Holt PG. Cytokine modulation of the immunosuppressive phenotype of pulmonary alveolar macrophage populations. Immunology. 1995;86(2):231-7.

56. Misharin AV et al. Monocyte-derived alveolar macrophages drive lung fibrosis and persist in the lung over the life span. J Exp Med. 2017;214(8):2387-404.

57. Landsman $L$ et al. Distinct differentiation potential of blood monocyte subsets in the lung. $J$ Immunol. 2007;178(4):2000-7.

58. Stumbles PA et al. Resting respiratory tract dendritic cells preferentially stimulate $\mathrm{T}$ helper cell type 2 (Th2) responses and require obligatory cytokine signals for induction of Th1 immunity. J Exp Med 1998;188(11):2019-31.

59. Lambrecht BN et al. Dendritic cells are required for the development of chronic eosinophilic airway inflammation in response to inhaled antigen in sensitized mice. J Immunol. 1998;160(8):4090-7.

60. Demedts IK et al. Identification and characterization of human pulmonary dendritic cells. Am J Respir Cell Mol Biol. 2005;32(3):177-84

61. Schlecht $\mathrm{G}$ et al. Murine plasmacytoid dendritic cells induce effector/ memory CD8+ T-cell responses in vivo after viral stimulation. Blood. 2004;104(6):1808-15.

62. Holt PG et al. Origin and steady-state turnover of Class II MHC-bearing dendritic cells in the epithelium of the conducting airways. J Immunol. 1994;153(1):256-61.

63. Neyt K, Lambrecht BN. The role of lung dendritic cell subsets in immunity to respiratory viruses. Immunol Rev. 2013;255(1):57-67.

64. Guilliams M et al. Dendritic cells, monocytes and macrophages: a unified nomenclature based on ontogeny. Nat Rev Immunol. 2014;14(8):571-8.

65. Ballesteros-Tato A et al. Temporal changes in dendritic cell subsets, cross-priming and costimulation via CD70 control CD8(+) T cell responses to influenza. Nat Immunol. 2010;11(3):216-24.

66. Martin-Gayo E et al. Plasmacytoid dendritic cells resident in human thymus drive natural Treg cell development. Blood. 2010;115(26):5366-75.

67. de Heer HJ et al. Essential role of lung plasmacytoid dendritic cells in preventing asthmatic reactions to harmless inhaled antigen. J Exp Med. 2004:200(1):89-98.
68. Kool $\mathrm{M}$ et al. An anti-inflammatory role for plasmacytoid dendritic cells in allergic airway inflammation. J Immunol. 2009;183(2):1074-82.

69. Demedts IK et al. Different roles for human lung dendritic cell subsets in pulmonary immune defense mechanisms. Am J Respir Cell Mol Biol. 2006;35(3):387-93.

70. Masten BJ et al. Characterization of myeloid and plasmacytoid dendritic cells in human lung. $\mathrm{J} \mathrm{Immunol.}$ 2006;177(11):7784-93.

71. Asselin-Paturel $C$ et al. Mouse Type I IFN-producing cells are immature APCs with plasmacytoid morphology. Nat Immunol. 2001;2(12):1144-50.

72. Hornung $\vee$ et al. Replicationdependent potent IFN-alpha induction in human plasmacytoid dendritic cells by a singlestranded RNA virus. J Immunol. 2004;173(10):5935-43

73. Lund JM et al. Recognition of singlestranded RNA viruses by Toll-like receptor 7. Proc Natl Acad Sci U S A. 2004;101(15):5598-603.

74. Teijaro JR et al. Cutting edge: tissue-retentive lung memory CD4 T cells mediate optimal protection to respiratory virus infection. J Immunol. 2011;187(11):5510-4.

75. Turner DL et al. Lung niches for the generation and maintenance of tissue-resident memory $T$ cells. Mucosal Immunol. 2014;7(3):501-10.

76. Masopust D, Soerens AG. TissueResident $T$ Cells and Other Resident Leukocytes. Annu Rev Immunol. 2019;37:521-46.

77. Szabo PA et al. Location, location, location: tissue resident memory $T$ cells in mice and humans. Sci Immunol. 2019;4(34):eaas9673.

78. Park CO, Kupper TS. The emerging role of resident memory $T$ cells in protective immunity and inflammatory disease. Nat Med. 2015;21(7):688-97.

79. Purwar R et al. Resident memory $T$ cells $(T(R M))$ are abundant in human lung: diversity, function, and antigen specificity. PLoS One. 2011;6(1):e16245.

80. Sathaliyawala T et al. Distribution and compartmentalization of human circulating and tissue-resident memory T cell subsets. Immunity. 2013;38(1):187-97.

81. Jozwik A et al. RSV-specific airway resident memory CD8+ T cells and differential disease severity after experimental human infection. Nat Commun. 2015:6:10224.

82. $\mathrm{Wu} T$ et al. Lung-resident memory CD8 T cells (TRM) are indispensable for optimal cross-protection against pulmonary virus infection. J Leukoc Biol. 2014:95(2):215-24. 
83. Zens KD et al. Vaccine-generated lung tissue-resident memory $T$ cells provide heterosubtypic protection to influenza infection. $\mathrm{JCl}$ Insight. 2016;1(10):e85832.

84. Hondowicz BD et al. Interleukin-2Dependent Allergen-Specific TissueResident Memory Cells Drive Asthma. Immunity. 2016;44(1):155-66.

85. Turner DL et al. biased generation and in situ activation of lung tissueresident memory CD4 $T$ cells in the pathogenesis of allergic asthma. J Immunol. 2018;200(5):1561-9.

86. Gollwitzer ES et al. Lung microbiota promotes tolerance to allergens in neonates via PD-L1. Nat Med. 2014;20(6):642-7.

87. Lloyd CM, Hawrylowicz CM. Regulatory T cells in asthma. Immunity. 2009;31(3):438-49.

88. Duan W, Croft M. Control of regulatory $T$ cells and airway tolerance by lung macrophages and dendritic cells. Ann Am Thorac Soc. 2014;11(Suppl 5):S306-13.

89. Soroosh $P$ et al. Lung-resident tissue macrophages generate Foxp3+ regulatory $T$ cells and promote airway tolerance. J Exp Med. 2013;210(4):775-88.

90. Kearley $\mathrm{J}$ et al. Resolution of airway inflammation and hyperreactivity after in vivo transfer of CD4+CD25+ regulatory $T$ cells is interleukin 10 dependent. J Exp Med. 2005;202(11):1539-47.

91. Chen Z, O'Shea JJ. Regulation of IL-17 production in human lymphocytes. Cytokine. 2008;41(2):71-8.

92. Weaver CT et al. Th17: an effector CD4 $T$ cell lineage with regulatory $T$ cell ties. Immunity. 2006;24 (6):677-88.

93. Diefenbach A et al. Development, differentiation, and diversity of innate lymphoid cells. Immunity. 2014;41(3):354-65.
94. Borger JG et al. Editorial: the role of innate lymphoid cells in mucosal immunity. Front Immunol. 2020;11:1233.

95. Tait Wojno ED, Artis D. Emerging concepts and future challenges in innate lymphoid cell biology. J Exp Med. 2016;213(11):2229-48.

96. Spits $\mathrm{H}$ et al. Innate lymphoid cells--a proposal for uniform nomenclature. Nat Rev Immunol. 2013;13(2):145-9.

97. Neill DR et al. Nuocytes represent a new innate effector leukocyte that mediates Type-2 immunity. Nature. 2010;464(7293):1367-70.

98. Gasteiger $\mathrm{G}$ et al. Tissue residency of innate lymphoid cells in lymphoid and nonlymphoid organs. Science. 2015;350(6263):981-5

99. Denney L et al. Pulmonary epithelia cell-derived cytokine TGF-beta1 is a critical cofactor for enhanced innate lymphoid cell function. Immunity. 2015;43(5):945-58.

100. Mohapatra A et al. Group 2 innate Iymphoid cells utilize the IRF4-IL-9 module to coordinate epithelial cell maintenance of lung homeostasis. Mucosal Immunol. 2016;9(1):275-86.

101. Ardain A et al. Type 3 ILCs in lung disease. Front Immunol. 2019;10:92.

102. Vantourout P, Hayday A. Six-ofthe-best: unique contributions of gammadelta $T$ cells to immunology. Nat Rev Immunol. 2013;13(2):88-100.

103. Sim GK et al. Homing and in situ differentiation of resident pulmonary Iymphocytes. Int Immunol. 1994;6(9):1287-95.

104. Paget $C$ et al. CD3bright signals on gammadelta $T$ cells identify IL-17Aproducing Vgamma6Vdelta1+ T cells. Immunol Cell Biol. 2015:93(2):198-212.

105. Wands JM et al. Distribution and leukocyte contacts of gammadelta $\mathrm{T}$ cells in the lung. J Leukoc Biol. 2005;78(5):1086-96
106. Cheng M, Hu S. Lung-resident gammadelta $T$ cells and their roles in lung diseases. Immunology. 2017:151(4):375-84.

107. Vanaudenaerde BM et al. Innate and adaptive interleukin-17-producing lymphocytes in chronic inflammatory lung disorders. Am J Respir Crit Care Med. 2011;183(8):977-86.

108. Simonian PL et al. IL-17A-expressing $T$ cells are essential for bacterial clearance in a murine model of hypersensitivity pneumonitis. J Immunol. 2009;182(10):6540-9.

109. Nanno $M$ et al. Exacerbating role of gammadelta $T$ cells in chronic colitis of T-cell receptor alpha mutant mice. Gastroenterology. 2008;134 (2):481-90.

110. Borger JG et al. The influence of innate lymphoid cells and unconventional $\mathrm{T}$ cells in chronic inflammatory lung disease. Front Immunol. 2019;10:1597.

111. Nurwidya F et al. The role of innate and adaptive immune cells in the immunopathogenesis of chronic obstructive pulmonary disease. Tuberc Respir Dis (Seoul). 2016;79(1):5-13

112. Holtzman MJ. Asthma as a chronic disease of the innate and adaptive immune systems responding to viruses and allergens. J Clin Invest. 2012;122(8):2741-8.

113. Iwasaki A et al. Early local immune defences in the respiratory tract. Nat Rev Immunol. 2017;17(1):7-20.

114. Gonzalez $\mathrm{H}$ et al. Roles of the immune system in cancer: from tumor initiation to metastatic progression. Genes Dev. 2018;32(19-20):1267-84.

115. Lugade AA et al. Cigarette smoke exposure exacerbates lung inflammation and compromises immunity to bacterial infection. J Immunol. 2014;192(11):5226-35.

116. Mehta $\mathrm{H}$ et al. Cigarette smoking and innate immunity. Inflamm Res. 2008;57(11):497-503. 